PROCEEDINGS OF THE

AMERICAN MATHEMATICAL SOCIETY

Volume 125, Number 12, December 1997, Pages 3447-3455

S 0002-9939(97)04249-4

\title{
A HYPERBOLIC-BY-HYPERBOLIC HYPERBOLIC GROUP
}

\author{
LEE MOSHER
}

(Communicated by Ronald M. Solomon)

\begin{abstract}
Given a short exact sequence of finitely generated groups$$
1 \rightarrow K \rightarrow G \rightarrow H \rightarrow 1
$$

it is known that if $K$ and $G$ are word hyperbolic, and if $K$ is nonelementary, then $H$ is word hyperbolic. In the original examples due to Thurston, as well as later examples due to Bestvina and Feighn, the group $H$ is elementary. We give a method for constructing examples where all three groups are nonelementary.
\end{abstract}

Given a short exact sequence of finitely generated groups

$$
1 \rightarrow K \rightarrow G \rightarrow H \rightarrow 1
$$

it is shown in [Mos96] that if $K$ and $G$ are word hyperbolic and $K$ is nonelementary (that is, not virtually cyclic), then $H$ is word hyperbolic.

Since the appearance of that result, several people have asked whether there is an example where all three groups are nonelementary. In all previously known examples the group $H$ is elementary. For instance, let $K=\pi_{1}(S)$ where $S$ is a closed hyperbolic surface, let $f: S \rightarrow S$ be a pseudo-Anosov homeomorphism, and let $M_{f}$ be the mapping torus of $f$, obtained from $S \times[0,1]$ by identifying $(x, 1) \sim(f(x), 0)$ for all $x \in S$. Thurston proved [Ota96] that $M_{f}$ is a hyperbolic 3 -manifold and so the group $G=\pi_{1}\left(M_{f}\right)$ is word hyperbolic. We therefore obtain a short exact sequence $1 \rightarrow \pi_{1}(S) \rightarrow \pi_{1}(M) \rightarrow \mathbf{Z} \rightarrow 1$. This was generalized by Bestvina and Feighn [BF92] who showed that if $K$ is any word hyperbolic group, and if $G$ is the extension of $K$ by a hyperbolic automorphism of $K$, then $G$ is word hyperbolic; we again obtain an example with $H=\mathbf{Z}$.

Here is a method for constructing examples where each of $K, G, H$ is nonelementary. We extend a hyperbolic surface group by a "large free group" of hyperbolic automorphisms, i.e. pseudo-Anosov mapping classes. Let $S$ be a closed hyperbolic surface, pick a base point $p \in S$, let $K=\pi_{1}(S, p)$ be the fundamental group, let $\mathcal{M C G}(S)$ be the mapping class group, and let $\mathcal{M C G}(S, p)$ be the mapping class group of $S$ punctured at $p$. Recall [Bir74] the following short exact sequences,

Received by the editors May 4, 1996.

1991 Mathematics Subject Classification. Primary 20F32; Secondary 57M07, 20F28.

Partially supported by NSF grant \# DMS-9204331.

(C)1997 American Mathematical Society 
which are isomorphic by the Dehn-Nielsen theorem [Sti87]:



The action of a pseudo-Anosov $\Phi \in \mathcal{M C G}(S)$ on the space of projective measured foliations $\mathcal{P} \mathcal{M F}(S)$ has a fixed point set $\operatorname{Fix}(\Phi)$ consisting of an attractor and a repeller; all other orbits go from the repeller to the attractor $\left[\mathrm{FLP}^{+} 79\right]$. A set $\mathcal{A}$ of pseudo-Anosov mapping classes is independent if the sets $\operatorname{Fix}(\Phi)$ are pairwise disjoint for $\Phi \in \mathcal{A}$. Equivalently, if $N(\Phi)$ is the largest virtually abelian subgroup of $\mathcal{M C G}(S)$ containing $\Phi$, then $N(\Phi) \cap N(\Psi)=\{1\}$ for $\Phi \neq \Psi \in \mathcal{A}$ (see [BLM83], proof of Theorem A).

Theorem. Let $S$ be a closed hyperbolic surface. Let $\Phi_{1}, \ldots, \Phi_{m} \in \mathcal{M C G}(S)$ be an independent set of pseudo-Anosov mapping classes of $S$. Let $i_{1}, \ldots, i_{m}$ be positive integers, and consider the subgroup $H=\left\langle\Phi_{1}^{i_{1}}, \ldots, \Phi_{m}^{i_{m}}\right\rangle$ of $\mathcal{M C G}(S)$. Let $G<$ $\mathcal{M C G}(S, p)$ be the preimage of $H$, so we have a short exact sequence

$$
1 \rightarrow K \rightarrow G \rightarrow H \rightarrow 1 .
$$

If $i_{1}, \ldots, i_{m}$ are sufficiently large, then the group $H$ is free on the given generators, and $G$ is word hyperbolic.

Remark. Independent sets of pseudo-Anosov mapping classes of any cardinality are easily constructed. Indeed, pseudo-Anosov fixed point sets are dense in the space $\mathcal{P} \mathcal{M F}(S) \times \mathcal{P} \mathcal{M F}(S)[$ Mas94].

Remark. Our proof makes heavy use of surface topology, in particular the stable and unstable laminations of pseudo-Anosov homeomorphisms. Bestvina, Feighn, and Handel give examples where $K$ is a free group [BFH96], by proving an analogue of the above theorem using their theory of stable and unstable laminations for irreducible automorphisms of free groups.

Remark. Normal hyperbolic subgroups of hyperbolic groups are studied by Mitra [Mit], where it is proved that the inclusion map $K \rightarrow G$ extends continuously to a map $K \cup \partial K \rightarrow G \cup \partial G$, where $\partial K$ and $\partial G$ are the Gromov boundaries, so that the image of $\partial K$ is all of $\partial G$. Mitra's theorem extends the result of Cannon-Thurston $[\mathrm{CT}]$ which applies to the case when $G$ is the fundamental group of a hyperbolic 3 -manifold fibering over the circle and $K$ is the fundamental group of the fiber.

Problem. Does the above theorem have a generalization when $K$ is replaced by an arbitrary word hyperbolic group? Rips and Sela have proved the existence of a JSJ decomposition for word hyperbolic groups [RS95]. As a consequence they can characterize those word hyperbolic groups $K$ for which $\operatorname{Out}(K)$ is infinite. Given such a group $K$, is there a good notion of irreducible automorphisms and their stable and unstable laminations?

Problem. Is there a way to classify those subgroups $H<\mathcal{M C G}(S)$ whose inverse image $G<\mathcal{M C G}(S, p)$ is word hyperbolic? Necessary conditions are that $H$ is word hyperbolic [Mos96], and except for the identity $H$ consists entirely of pseudoAnosov mapping classes (otherwise, $G$ has a $\mathbf{Z}^{2}$ subgroup). In particular, is there such a subgroup $H$ which is not virtually free? Analogous questions can be asked 
for subgroups of $\operatorname{Out}(K)$, where $K$ is any other word hyperbolic group such that $\operatorname{Out}(K)$ is infinite, for example a free group.

Proof of theorem. The statement that $H$ is free on the given generators, for sufficiently large exponents, is a consequence of the standard "Schottky" argument first used by Klein; see [McC85] for the argument applied to $\mathcal{M C G}(S)$ acting on $\mathcal{P} \mathcal{M F}(S)$.

The proof that $G$ is word hyperbolic will be an application of the methods of Bestvina \& Feighn [BF92], and thus we depend ultimately on the combination theorem of [BF92]. However, instead of quoting their combination theorem directly, we instead adapt their proof that a word hyperbolic group extended by a hyperbolic automorphism gives a word hyperbolic group.

For notational convenience, we give the proof for an independent pair of pseudoAnosov mapping classes $\Phi, \Psi \in \mathcal{M C G}(S)$. We shall freely use well known results about pseudo-Anosov homeomorphisms and their mapping classes; for references see $\left[\mathrm{FLP}^{+} 79\right]$, [BC88].

Choose pseudo-Anosov homeomorphisms $\phi, \psi$ on $S$ representing $\Phi, \Psi$. By taking powers, we may assume that $\phi$ and $\psi$ each have a fixed point on $S$, and we may assume that this fixed point is a pseudo-Anosov singularity. By isotopy, we may assume that the fixed point is $p$. Thus, we may regard $\phi, \psi$ as lying in $\mathcal{M C G}(S, p)$. Using the natural isomorphism $\mathcal{M C G}(S, p) \rightarrow \operatorname{Aut}(K)$, given $g \in \mathcal{M C G}(S, p)$ we use the same symbol $g$ to denote the corresponding element of $\operatorname{Aut}(K)$. Fix a generating set on $K$, with corresponding word metric $|\cdot|$.

Given $g \in \operatorname{Aut}(K)$ and $\lambda>0$, let $\operatorname{Stretch}_{g}^{\lambda}=\{x \in K|| g(x)|>\lambda| x \mid\}$. If $x \in \operatorname{Stretch}_{g}^{\lambda}$ we say that $g$ stretches $x$ by a factor of $\lambda$.

Lemma: Three out of four stretch. For any $\lambda>1$, if $m, n$ are sufficiently large integers, the following is true. For any nontrivial $x \in K$, at least three of the four elements $\left\{\phi^{m}, \phi^{-m}, \psi^{n}, \psi^{-n}\right\}$ stretch $x$ by a factor of $\lambda$.

For a set of $M$ independent pseudo-Anosovs, one would prove that " $2 M-1$ out of $2 M$ stretch". Before proving the lemma, we use it to complete the theorem.

Construct the double mapping torus $M=M_{\phi^{m}}, \psi^{n}$ by taking $S_{1}, S_{2}$ to be homeomorphic copies of $S$ via homeomorphisms $f_{i}: S_{i} \rightarrow S$, and taking $M$ to be the quotient space of $S_{1} \times[0,1] \cup S_{2} \times[0,1] \cup S$ by identifying $\left(x_{i}, 0\right) \sim f_{i}\left(x_{i}\right)$, $\left(x_{1}, 1\right) \sim \phi\left(f_{1}\left(x_{1}\right)\right)$, and $\left(x_{2}, 1\right) \sim \psi\left(f_{2}\left(x_{2}\right)\right)$, for $x_{1} \in S_{1}, x_{2} \in S_{2}$. The goal is to prove that the complex $M$ is hyperbolic, which just means that its fundamental group is word hyperbolic.

Now look at the proof in $\S 5$ of [BF92], that if $g: K \rightarrow K$ is a hyperbolic automorphism of a word hyperbolic group $K$, then the ordinary mapping torus of $g$ is hyperbolic. That proof quickly reduces to the following special case: if $x \in K$ then $\operatorname{Max}\left\{|g(x)|,\left|g^{-1}(x)\right|\right\} \geq 3|x|$. Rewording this statement, for any two elements $g_{1} \neq g_{2} \in\left\{g, g^{-1}\right\}$ and any $x \in K$, at least one of $g_{1}, g_{2}$ stretches $x$ by a factor of 3.

In our situation, the needed statement follows from the lemma Three out of four stretch. Namely, if $m, n$ are sufficiently large, then for any two elements $g_{1} \neq g_{2} \in$ $\left\{\phi^{m}, \phi^{-m}, \psi^{n}, \psi^{-n}\right\}$ and any $x \in K$, at least one of $g_{1}, g_{2}$ stretches $x$ by a factor of 3. Now follow the proof in [BF92] to show that $M_{\phi^{m}}, \psi^{n}$ is hyperbolic.

Proof of lemma: Three out of four stretch. Consider a pseudo-Anosov homeomorphism $g: S \rightarrow S$ with stable and unstable measured foliations $f_{g}^{s}, f_{g}^{u}\left[\mathrm{FLP}^{+} 79\right]$. We 
use the conventions of dynamical systems, where $g$ stretches the leaves of $f_{g}^{u}$ and contracts the leaves of $f_{g}^{s}$ by the same factor $\lambda_{g}>1$. Recall that the Fubini product of the transverse measures on $f_{g}^{s}$ and $f_{g}^{u}$ defines a singular Euclidean structure on $S$, with isolated cone singularities of angles $2 \pi n$ for $n \geq 3$. Away from these " $g$-singularities", local Eulidean coordinates are uniquely determined up to translation and rotation through $180^{\circ}$ so that $f_{g}^{s}$ measures $|d y|$ and $f_{g}^{u}$ measures $|d x|$; hence $f_{g}^{s}$ has horizontal leaves and $f_{g}^{u}$ has vertical leaves. The Euclidean structure determines a metric $d_{g}$ on $S$ for which each path can be homotoped rel endpoints to a unique geodesic. Let $c_{g}(x)$ be the based geodesic representing $x$. We may assume that the metrics $d_{g}, d_{g^{-1}}$ are identical.

Lift the metrics $d_{\Phi}, d_{\Psi}$ to metrics $\tilde{d}_{\Phi}, \tilde{d}_{\Psi}$ on the universal cover $\tilde{S}$. Choose a base point $\tilde{p} \in \tilde{S}$ lifting $p$. Identifying $K$ with the orbit of $\tilde{p}$ under $K$, we have three left invariant metrics on $K$ : the word metric, and the restrictions of $\tilde{d}_{\Phi}, \tilde{d}_{\Psi}$. These metrics are all Lipschitz equivalent to each other (see [Mil68] and also [Can91]). Let $C \geq 1$ be a Lipschitz constant for any two of them, i.e. the ratio of any two of the metrics is bounded by $C$.

Now let Stretch ${ }_{g}^{\lambda}$ be the set of $x \in K$ such that $g$ stretches $x$ by at least $\lambda$ when measured in the metric $\tilde{d}_{g}$. Note that Stretch ${ }_{g}^{\prime} \subset \operatorname{Stretch}_{g}^{\lambda / C^{2}}$.

If $0<\eta<1$, define Slope $g_{g}^{\eta}$ to be the set of all $x \in K$ such that the (unsigned) Euclidean angle between $c_{g}(x)$ and $f_{g}^{s}$ is at least $\eta$, on a subset of $c_{g}(x)$ of length at least $\eta$. Length $c_{g}(x)$. Then if $n$ is sufficiently large, Slope ${ }_{g}^{\eta} \subset \operatorname{Stretch}_{g^{n}}{ }^{n}$; just choose $n$ so large that if the vector $v \in \mathbf{E}^{2}$ makes an angle at least $\eta$ with the horizontal axis, then the matrix

$$
\left(\begin{array}{cc}
\lambda_{g}^{-n} & 0 \\
0 & \lambda_{g}^{n}
\end{array}\right)
$$

stretches $v$ by a factor of at least $\lambda / \eta$.

We would like to compare stretching properties of different pseudo-Anosov homeomorphisms, but the Euclidean coordinates systems are different. To make a common coordinate system, fix a hyperbolic structure on $S$. The measured foliations $f_{g}^{s}, f_{g}^{u}$ can be straightened to measured geodesic laminations $\ell_{g}^{s}, \ell_{g}^{u}$ [BC88]. There is a map $q: S \rightarrow S$, homotopic to the identity, such that the point preimages are of three kinds: the closure of a component of $S-\left(\ell_{g}^{s} \cup \ell_{g}^{u}\right)$, which is always a polygon with $2 k$-sides for some $k \geq 2$; the closure of a component of $\ell_{g}^{s}-\ell_{g}^{u}$ or of $\ell_{g}^{u}-\ell_{g}^{s}$; or a point of $\ell_{g}^{s} \cap \ell_{g}^{u}$. This sets up a 1-1 correspondence between leaves of $\ell_{g}^{s}$ and "smooth" leaves of $f_{g}^{s}$, where a smooth leaf is either a nonsingular leaf or the union of two singular half-leaves meeting at a $g$-singularity at an angle of $180^{\circ}$. There is a similar $1-1$ correspondence for $\ell_{g}^{u}$ and $f_{g}^{u}$. Also, the polygons with $k \geq 3$ are in 1-1 correspondence with $g$-singularities.

Let $c(x)$ be the based hyperbolic geodesic representing $x \in K$. To say that $x \notin \operatorname{Slope}_{g}^{\eta}$ says that $c_{g}(x)$ is roughly parallel to $f_{g}^{s}$ for most of the length of $c_{g}(x)$. From this, we want to conclude that $c(x)$ is roughly parallel to $\ell_{g}^{s}$ for most of the length of $c(x)$. This is intuitively obvious; here is a careful formulation of what we need.

Let $\mathbf{P} S$ be the projectivization of the tangent vector bundle of $S$, also known as the tangent line bundle. Each geodesic lamination lifts to $\mathbf{P} S$, and we use the same symbol for the lift. 
Sublemma: Parallel corresponds. Given a pseudo-Anosov $g$ and $0<\epsilon<1$, there exists $0<\eta<1$ such that if $x \in K-$ Slope $_{g}^{\eta}$, then on a subset of $c_{x}$ of length at least $(1-\epsilon) \operatorname{Length}\left(c_{x}\right)$, the distance between the tangent line of $c_{x}$ and the set $\ell_{g}^{s}$, measured in $\mathbf{P} S$, is at most $\epsilon$.

The proof we offer below is somewhat technical. Taking this on faith for the moment, we use it to complete the proof that Three out of four stretch.

Choose $\epsilon \in(0,1 / 2)$ so small that the laminations $\ell_{\phi}^{s}, \ell_{\phi^{-1}}^{s}=\ell_{\phi}^{u}, \ell_{\psi}^{s}, \ell_{\psi^{-1}}^{s}=\ell_{\psi}^{u}$ are all at distance at least $2 \epsilon$ from each other in $\mathbf{P} S$; this is possible because these laminations, lifted to $\mathbf{P} S$, are pairwise disjoint closed sets. Choose $\eta \in(0,1)$ so that the conclusions of the above lemma hold for each $g \in\left\{\phi, \phi^{-1}, \psi, \psi^{-1}\right\}$. Since $1-\epsilon>1 / 2$ it follows that $K-\operatorname{Slope}_{\phi}^{\eta}, K-\operatorname{Slope}_{\phi^{-1}}^{\eta}, K-\operatorname{Slope}_{\psi}^{\eta}, K-$ Slope $_{\psi^{-1}}^{\eta}$ are pairwise disjoint subsets of $K$. Therefore, each $x \in K$ lies in at least three out of the four sets $\operatorname{Slope}_{\phi}^{\eta}$, $\operatorname{Slope}_{\phi^{-1}}^{\eta}, \operatorname{Slope}_{\psi}^{\eta}$, $\operatorname{Slope}_{\psi^{-1}}^{\eta}$. Now choose $m, n$ so large that $\operatorname{Slope}_{\Phi}^{\eta} \subset \operatorname{Stretch}^{\prime}{ }_{\Phi^{m}}{ }^{2} \lambda$, and similarly for $\Phi^{-m}, \Psi^{n}, \Psi^{-n}$. Hence Slope $_{\Phi}^{\eta} \subset$ Stretch $_{\Phi^{m}}^{\lambda}$ etc., so each $x \in K$ lies in at least three out of the four sets $\operatorname{Stretch}_{\Phi^{m}}^{\lambda}, \operatorname{Stretch}_{\Phi^{-m}}^{\lambda}, \operatorname{Stretch}_{\Psi^{n}}^{\lambda}, \operatorname{Stretch}_{\Psi^{-n}}^{\lambda}$.

Proof of sublemma: Parallel corresponds. The first step is to find very long segments $\alpha_{i} \subset c_{g}(x)$ and leaf segments $\beta_{i}$ of $f_{g}^{s}$ such that the $\alpha_{i}$ cover most of $c_{g}(x)$, and $\alpha_{i}$ is homotopic to $\beta_{i}$ by a homotopy moving points along short paths. Then we want to find corresponding hyperbolic segments $\alpha_{i}^{\prime} \subset c(x)$ and $\beta_{i}^{\prime} \subset \ell_{g}^{s}$, so that $\alpha_{i}^{\prime}, \beta_{i}^{\prime}$ are homotopic moving the endpoints along short paths. Defining the corresponding segments will require constructing gridworks in the surface which allow comparisons between the singular Euclidean geometry and the hyperbolic geometry. Finally, from hyperbolic geometry it follows that for most of the length of $\alpha_{i}^{\prime}$, the tangent line to $\alpha_{i}^{\prime}$ is very close to a tangent line of $\beta_{i}^{\prime}$ in $\mathbf{P} S$.

Since $x \in K-\operatorname{Slope}_{g}^{\eta}$, on a subset of $c_{g}(x)$ of length at least $(1-\eta)$ Length $c_{g}(x)$, the angle between $c_{g}(x)$ and $f_{g}^{s}$ is at most $\eta$. Here is a method for locating long segments of $c_{g}(x)$, where the angle is at most $\eta$, and which are homotopic to leaf segments of $f_{g}^{s}$ via a short homotopy.

Define an $\eta$-lever to be a homotopy from a $d_{g}$-geodesic segment $\alpha$ to a horizontal segment $\beta$, such that each track of the homotopy is a reparameterized vertical geodesic segment, possibly degenerate, the track of each point of int $(\alpha)$ is disjoint from $g$-singularities, and $\operatorname{int}(\alpha)$ makes an angle at most $\eta$ with the horizontal. A lever is denoted $(\alpha, \beta), \alpha$ is called the inclined edge of the lever, and $\beta$ is called the horizontal edge of the lever. Note that a lever is maximal if and only if the track of each endpoint of $\alpha$ contains a $g$-singularity. The length of the lever is $\operatorname{Length}(\alpha)$, which is at least as large as Length $(\beta)$. The height of the lever is the maximum length of the tracks of the points of $\alpha$, which is achieved at the endpoints. Given a geodesic path between $g$-singularities whose interior is disjoint from $g$-singularities and has slope at most $\eta$, then it is easy to find maximal $\eta$-levers whose inclined edges cover the path.

We claim that for each $L, H>0$ there exists $\eta=\eta(L, H)>0$ such that every maximal $\eta$-lever has length at least $L$ and height at most $H$; this gives our first condition on $\eta$, where $L$ and $H$ are to be determined later.

To obtain the upper bound on height, let $\sigma$ be the union of all vertical segments of length $H / 4$ based at a singularity, and note that there is an upper bound $C_{0}$ to the length of any horizontal segment disjoint from $\sigma$. Then any vertical segment 
of height $H / 2$ can be homotoped horizontally a distance at most $C_{0}$ before its midpoint encounters $\sigma$; hence one of its points must encounter a $g$-singularity. So if an $\eta$-lever has a vertical track of length $H / 2$, then the longer vertical tracks beyond it cannot have height greater than $H / 2+\eta C_{0}$. Therefore if $\eta<H / 2 C_{0}$ then the height of an $\eta$-lever is at most $H$. We are free to choose $H$ at will, and we choose $H=1$.

If the lower bound on length does not hold, we can choose a sequence $\eta_{n} \rightarrow 0$ and find maximal $\eta_{n}$-levers in $S$ of length less than $L$ and height less than $\eta_{n} \cdot L \rightarrow 0$. As $n \rightarrow \infty$, these levers have a subsequence converging to a segment of $f_{g}^{s}$ with endpoints at $g$-singularities. Such a segment must be degenerate. Thus, as $n \rightarrow \infty$ the lengths of the $\eta_{n}$-levers go to zero, and since $\eta_{n} \rightarrow 0$ we obtain geodesic paths between $g$-singularities of arbitrarily small length. That is absurd.

We may now find $\eta$-levers in $S$ of length at least $L$ and height at most $H=1$, with inclined segments $\alpha_{i}$ and horizontal segments $\beta_{i}$, so that the $\alpha_{i}$ cover at least $1-\eta$ of the total length of $c_{g}(x)$.

Now we prepare for finding corresponding hyperbolic geodesic segments. To each $k$-pronged $g$-singularity $s$, there corresponds a $2 k$-gon component of $S-\left(f_{g}^{s} \cup f_{g}^{u}\right)$; pick a point $s^{\prime}$ in this component. This correspondence is such that there is a canonical path homotopy class $\rho_{s s^{\prime}}$ from $s$ to $s^{\prime}$. Define a singular connector $\gamma$ to be a $d_{g}$-geodesic segment in $S$ whose endpoints lie on singularities; hence there corresponds to $\gamma$ a hyperbolic geodesic segment $\gamma^{\prime}$ : if $\gamma$ joins $s_{1}$ to $s_{2}$ then $\gamma^{\prime}$ joins $s_{1}^{\prime}$ to $s_{2}^{\prime}$, and $\gamma^{\prime}$ is determined by the condition that $\gamma * \rho_{s_{2} s_{2}^{\prime}} * \gamma^{\prime-1} * \rho_{s_{1} s_{1}^{\prime}}^{-1}$ is homotopically trivial.

We claim that intersections between singular connectors are preserved, when passing from the Euclidean setting to the hyperbolic setting, in the following sense. Suppose that $\gamma_{i}$ are singular connectors for $i=1,2$, and for simplicity we assume that $\gamma_{1}$ has no interior singularities. Suppose that $\tilde{\gamma}_{i}$ is a lift of $\gamma_{i}, \tilde{\gamma}_{i}^{\prime}$ is the corresponding lift of $\gamma_{i}^{\prime}, \partial \tilde{\gamma}_{1} \cap \partial \tilde{\gamma}_{2}=\emptyset$, and $\tilde{\gamma}_{1} \cap \tilde{\gamma}_{2} \neq \emptyset$. Then either $\tilde{\gamma}_{1}^{\prime} \cap \tilde{\gamma}_{2}^{\prime} \neq \emptyset$ or $\tilde{\gamma}_{2}^{\prime}$ comes within a bounded distance of an endpoint of $\tilde{\gamma}_{1}^{\prime}$.

To prove the claim, we use a convexity argument. Let $\partial \tilde{\gamma}_{i}=\left\{v_{i}, w_{i}\right\}$. Each $k$-pronged $\tilde{g}$-singularity $s \in \tilde{S}$ divides $\tilde{S}$ into $2 k$ quadrants, each quadrant bounded by a horizontal and vertical half-leaf with endpoint at $s$. Any geodesic segment missing $s$ must stay in some consecutive sequence of at most three quadrants at $s$, and since $k \geq 3$ the union of these quadrants is a convex subset of $\tilde{S}$. Let $A$ (resp. $B$ ) be the union of quadrants of $v_{1}$ (resp. $w_{1}$ ) that $\tilde{\gamma}_{2}$ intersects. Therefore $\tilde{\gamma}_{2}$ is contained in the convex set $D=A \cap B$, which has two boundary components, each the union of a stable half-leaf and an unstable half-leaf joined at a corner (see Figure 1). Now $\tilde{\gamma}_{1}$ is the geodesic connecting the corners of $D$, and the geodesic $\tilde{\gamma}_{2}$ crosses $\tilde{\gamma}_{1}$. Therefore, $v_{2}, w_{2}$ are contained in opposite components of $D-\tilde{\gamma}_{1}$. Corresponding to $D$ is a hyperbolic convex set $D^{\prime}$; if a boundary component of $D$ consists of half-leaves $l^{s}$ and $l^{u}$ meeting at a $k$-pronged singularity $s$, then the corresponding boundary component of $D^{\prime}$ consists of half-leaves $l^{\prime s}$ and $l^{\prime u}$, and if $k \geq 4$ the boundary component contains $2 k-6$ additional sides of the $2 k$-gon corresponding to $s$. The geodesic $\tilde{\gamma}_{1}^{\prime}$, when enlarged a bounded amount at each end, forms a geodesic $\tilde{\gamma}_{1}^{\prime \prime}$ cutting $D^{\prime}$ into two components. Since $\tilde{\gamma}_{1}$ has no interior singularities, then two singularities in $D$ lie in the same component of $D-\tilde{\gamma}_{1}$ if and only if the corresponding point lies in the same component of $D^{\prime}-\tilde{\gamma}_{1}^{\prime \prime}$; the point is that under the collapsing map $\tilde{q}: \tilde{S} \rightarrow \tilde{S}$, the path $\tilde{q} \circ \tilde{\gamma}_{1}^{\prime \prime}$ is homotopic in $D$ to $\tilde{\gamma}_{1}$, 



FIGURE 1

via a homotopy which is disjoint from any $\tilde{g}$-singularities except for the corners of $D$. The points $v_{2}^{\prime}, w_{2}^{\prime}$ are therefore contained in opposite components of $D^{\prime}-\tilde{\gamma}_{1}^{\prime \prime}$, so $\tilde{\gamma}_{2}^{\prime} \cap \tilde{\gamma}_{1}^{\prime \prime} \neq \emptyset$. It follows that $\tilde{\gamma}_{2}^{\prime} \cap \tilde{\gamma}_{1}^{\prime} \neq \emptyset$ or $\tilde{\gamma}_{2}^{\prime}$ comes within a bounded distance of $\partial \tilde{\gamma}_{1}^{\prime}$, establishing the claim.

Also, if we assume that $\tilde{\gamma}_{1} \cap \tilde{\gamma}_{2}$ consists of a common boundary point, then the same is true of $\tilde{\gamma}_{1}^{\prime} \cap \tilde{\gamma}_{2}^{\prime}$, by an easier argument.

Construct a cell-decomposition $\mathcal{C}$ of $S$ whose vertices are the $g$-singularities $s$, and whose edges $\gamma$ are singular connectors. This can be achieved by the Delauney construction: define a spine for $S-\{g$-singularities $\}$ consisting of those points whose closest $g$-singularity is not unique; then take the dual cell complex. By the previous paragraph, we obtain a corresponding cell-decomposition $\mathcal{C}^{\prime}$ of $S$, whose vertices are the $s^{\prime}$ and whose edges are the $\gamma^{\prime}$.

Now we return to the collection of maximal $\eta$-levers $\left(\alpha_{i}, \beta_{i}\right)$, whose inclined segments $\alpha_{i}$ cover at least $1-\eta$ of the length of $c_{g}(x)$. The complexes $\mathcal{C}$ and $\mathcal{C}^{\prime}$ are the "euclidean and hyperbolic gridworks" which we now use to define a subsegment $\alpha_{i}^{\prime}$ of $c(x)$ corresponding to $\alpha_{i}$.

Choose a lift $\tilde{c}_{g}(x)$, and find a maximal subsegment contained in $\tilde{\alpha}_{i}$ with endpoints in the 1-skeleton of $\tilde{\mathcal{C}}$. An endpoint of this subsegment lies on an edge $\tilde{\gamma}$ of $\tilde{\mathcal{C}}$, and by the above claim there is a corresponding point on $\tilde{c}(x)$ which is the closest point to the edge $\tilde{\gamma}^{\prime}$ of $\tilde{\mathcal{C}}^{\prime}$; take this to be the corresponding endpoint of $\tilde{\alpha}_{i}^{\prime}$. This finishes the definition of $\alpha_{i}^{\prime}$.

We now obtain our second condition on $\eta$ : since all the metrics involved are quasi-isometric, it follows that for any $0<\epsilon<1$ we may choose $0<\eta<1$ so that for any $x \in K-$ Slope $_{g}^{\eta}$, the $\alpha_{i}^{\prime}$ cover at least $\sqrt{1-\epsilon}$ of the length of $c(x)$.

The lever $\left(\alpha_{i}, \beta_{i}\right)$ determines a singular connector $\gamma_{i}$ in the obvious manner, using the fact that the tracks of the endpoints of $\alpha_{i}$ hit $g$-singularities. We thus obtain a corresponding hyperbolic segment $\gamma_{i}^{\prime}$, and there is a homotopy between $\alpha_{i}^{\prime}$ and $\gamma_{i}^{\prime}$ moving endpoints through tracks of length at most $C_{1}$, where $C_{1}$ depends only on $\mathcal{C}$. Now $\gamma_{i}^{\prime}$ may be shortened to a maximal subsegment whose endpoints lie on $\ell_{g}^{u}$, and this subsegment may be homotoped through geodesics with endpoints on the same leaves of $\ell_{g}^{u}$, reaching a segment $\beta_{i}^{\prime}$ of $\ell_{g}^{s}$. The homotopy from $\gamma_{i}^{\prime}$ to $\beta_{i}^{\prime}$ moves endpoints a bounded distance at most $C_{2}$, where $C_{2}$ depends only on $\mathcal{C}, \ell_{g}^{s}$, $\ell_{g}^{u}$, and $H=1$. 
Using the fact that all the metrics involved are quasi-isometric [Can91], for each constant $L^{\prime}>0$ there exists a constant $L=L\left(L^{\prime}\right)$, such that if the lengths of all the levers are at least $L$ then the length of each $\alpha_{i}^{\prime}$ is at least $L^{\prime}$. Since the endpoints of $\alpha_{i}^{\prime}$ are moved at most $C_{1}+C_{2}$ under the homotopy to $\beta_{i}^{\prime}$, it follows that for most of the length of $\alpha_{i}^{\prime}$, the tangent vector is close to a tangent vector of $\beta_{i}^{\prime}$ in $\mathbf{P} S$. More precisely, given $\epsilon>0$ there exists $L^{\prime}=L^{\prime}\left(\epsilon, C_{1}, C_{2}\right)$ such that if $\alpha_{i}^{\prime}$ has length at least $L^{\prime}$ then on at least $\sqrt{1-\epsilon}$ of the length of $\alpha_{i}^{\prime}$, the tangent vector is within distance $\epsilon$ of a tangent vector of $\beta_{i}^{\prime}$. Therefore, on at least $1-\epsilon$ of the length of $c(x)$, the tangent line is within distance $\epsilon$ of a tangent vector to $\ell_{g}^{s}$.

This completes the proof of the lemma Parallel corresponds. To recap: given $\epsilon$, we have the "second condition" on $\eta$, determined by the requirement that if the $\alpha_{i}$ cover at least $1-\eta$ of $c_{g}(x)$, then the $\alpha_{i}^{\prime}$ cover at least $\sqrt{1-\epsilon}$ of $c(x)$. Also, $\epsilon$ together with $C_{1}$ and $C_{2}$ determines $L^{\prime}$, and $L^{\prime}$ determines $L$; and then $L$ and $H=1$ determine the "first condition" on $\eta$ so that each maximal $\eta$-lever has length at least $L$ and height at most $H=1$. If $\eta$ satisfies both conditions, and if $x \in K-$ Slope $_{g}^{\eta}$, then the tangent vector of $c(x)$ is within $\epsilon$ of a tangent vector to $\ell_{g}^{s}$, for at least $1-\epsilon$ of the length of $c(x)$.

\section{ACKNOWLEDGEMENTS}

I would like to thank Mark Feighn for several conversations clarifying the need for the lemma Three out of four stretch.

\section{REFERENCES}

[BC88] S. Bleiler and A. Casson, Automorphisms of surfaces after Nielsen and Thurston, LMS Student Texts, vol. 9, Cambridge University Press, 1988. MR 89k:57025

[BF92] M. Bestvina and M. Feighn, A combination theorem for negatively curved groups, J. Diff. Geom. 35 (1992), no. 1, 85-101. MR 93d:53053

[BFH96] M. Bestvina, M. Feighn, and M. Handel, Laminations, trees, and irreducible automorphisms of free groups, Geometric and Functional Analysis (1997), to appear.

[Bir74] J. Birman, Braids, links, and mapping class groups, Annals of Math. Studies, vol. 82, Princeton University Press, 1974. MR 51:11477

[BLM83] J. Birman, A. Lubotsky, and J. McCarthy, Abelian and solvable subgroups of the mapping class group, Duke Math. J. 50 (1983), no. 4, 1107-1120. MR 85k:20126

[Can91] J. Cannon, The theory of negatively curved spaces and groups, Ergodic theory, symbolic dynamics, and hyperbolic spaces (C. Series T. Bedford, M. Keane, ed.), Oxford Univ. Press, 1991. CMP 92:02

[CT] J. Cannon and W. P. Thurston, Group invariant Peano curves, preprint.

$\left[\mathrm{FLP}^{+} 79\right]$ A. Fathi, F. Laudenbach, V. Poenaru, et al., Travaux de Thurston sur les surfaces, Astérisque, vol. 66-67, Société Mathématique de France, Paris, 1979. MR 82m:57003

[Mas94] H. Masur, 1994, private correspondence.

[McC85] J. McCarthy, A "Tits-alternative" for subgroups of surface mapping class groups, Trans. AMS 291 (1985), no. 2, 583-612. MR 87f:57011

[Mil68] J. Milnor, A note on curvature and fundamental group, J. Diff. Geom. 2 (1968), 1-7. MR 38:636

[Mit] M. Mitra, Cannon-Thurston maps for hyperbolic group extensions, Topology (1997), to appear.

[Mos96] L. Mosher, Hyperbolic extensions of groups, J. Pure and Appl. Alg. 110 (1996), no. 3, 305-314. MR 97c:20056

[Ota96] J.-P. Otal, Le théorème d'hyperbolisation pour les variétés fibrées de dimension 3, Astérisque, no. 235, Société mathématique de france, 1996. MR 97e:57013 
[RS95] E. Rips and Z. Sela, Cyclic splittings of finitely presented groups and the canonical JSJ decomposition, Annals of Math. (1997), to appear.

[Sti87] J. Stillwell, The Dehn-Nielsen theorem, Papers on group theory and topology, by M. Dehn (J. Stillwell transl.), Springer, 1987.

Department of Mathematics and Computer Science, Rutgers University, Newark, New Jersey 07102

E-mail address: mosher@andromeda.rutgers.edu 